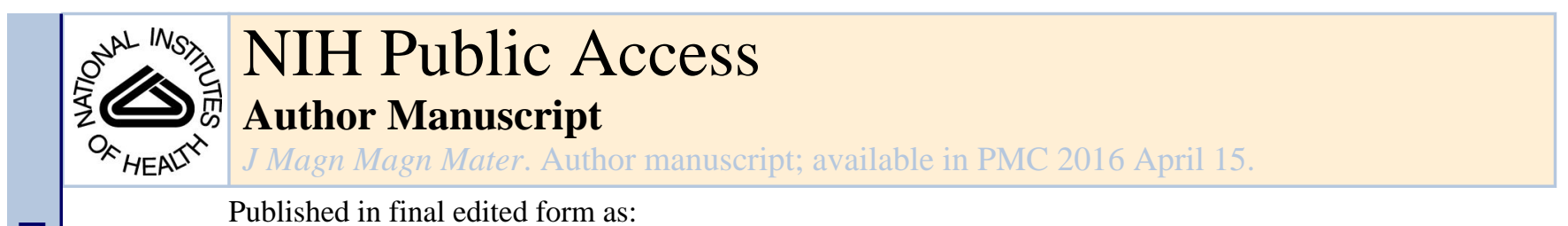

Published in final edited form as:

J Magn Magn Mater. 2015 April 15; 380: 295-298. doi:10.1016/j.jmmm.2014.09.059.

\title{
Analysis of Driven Nanorod Transport Through a Biopolymer Matrix
}

\author{
Lamar O. Mair ${ }^{1}{ }^{*}$, Irving N. Weinberg ${ }^{1}$, Alek Nacev ${ }^{1}$, Mario G. Urdaneta ${ }^{1}$, Pavel Stepanov ${ }^{1}$, \\ Ryan Hilaman ${ }^{1}$, Stephanie Himelfarb ${ }^{1}$, and Richard Superfine ${ }^{2}$ \\ ${ }^{1}$ Weinberg Medical Physics LLC, Bethesda, MD 20817 USA \\ ${ }^{2}$ Department of Physics and Astronomy, University of North Carolina, Chapel Hill, NC 27599 USA
}

\begin{abstract}
Applying magnetic fields to guide and retain drug-loaded magnetic particles in vivo has been proposed as a way of treating illnesses. Largely, these efforts have been targeted at tumors. One significant barrier to long range transport within tumors is the extracellular matrix (ECM). We perform single particle measurements of $18 \mathrm{~nm}$ diameter nanorods undergoing magnetophoresis through ECM, and analyze the motion of these nanorods in two dimensions. We observe intraparticle magnetophoresis in this viscoelastic environment and measure the fraction of time these nanorods spend effectively hindered, versus effectively translating.
\end{abstract}

\section{Keywords}

magnetophoresis; magnetic nanorods; particle tracking; extracellular matrix

\section{Introduction}

Magnetic micro- and nanoscale particles have proven useful in a variety of applications, including microfluidics [1], gene transfection [2], hyperthermia [3], drug delivery [4]-[7], and mechanically induced gene activation [8]. Magnetic fields have been used to achieve translational [9]-[12] and rotational manipulation [13] [15] of nanoparticles in a variety of environments and to various ends, and some groups have achieved elaborate control over particles with many degrees of freedom [16]. Implicit in the application and manipulation of magnetic nanoparticles is their interaction with the surrounding medium. This medium may be a Newtonian fluid or a complex non-Newtonian biopolymer system. In Newtonian solutions, particle motion during magnetophoresis is a composite of magnetic forces $\left(F_{\text {magnetic }}\right)$ and fluidic drag forces $\left(F_{\text {drag }}\right)$. In non-Newtonian environments the matrix can impose additional steric forces $\left(F_{\text {steric }}\right)$ and nonspecific surface adhesion $\left(F_{\text {surface }}\right)$ forces

() 2014 Elsevier B.V. All rights reserved.

*Correspondence: Lamar.Mair@gmail.com.

Publisher's Disclaimer: This is a PDF file of an unedited manuscript that has been accepted for publication. As a service to our customers we are providing this early version of the manuscript. The manuscript will undergo copyediting, typesetting, and review of the resulting proof before it is published in its final citable form. Please note that during the production process errors may be discovered which could affect the content, and all legal disclaimers that apply to the journal pertain. 
which can significantly complicate, and in many cases hinder, long range transport [17] [20].

An understanding of nanoparticle interactions with and transport through complex biopolymers is important for optimizing magnetically guided nanoparticle motion through tissues. Observing particle motion at the single particle level is one approach to understanding nanoparticle magnetophoresis. Using this method, we previously demonstrated significant differences in the motion of $18 \mathrm{~nm}$ diameter nanorods and larger diameter nanorods (55 nm and $200 \mathrm{~nm}$ ) [21]. Here we expand on our previous work by focusing on the dynamics of $18 \mathrm{~nm}$ diameter nanorod motion through ECM. We calculate average residence time as a fraction of overall experiment duration, and observe intra-pair magnetophoresis events. An important aspect of the work presented here is the fact that the components which make up the ECM meshwork are very similar to the diameters of the nickel nanowires used in these experiments [22]. Scanning electron micrographs of Matrigel and the as-grown nickel nanorods are shown in Fig. 1 for comparison.

Matrigel is a commercially available complex composed primarily of laminin, collagen IV, entactin, and heparin sulfate proteoglycans. Matrigel was chosen as a substitute for ex vivo extracellular matrix due to its ease of preparation and relatively high sample-to-sample homogeneity. As a multicomponent matrix, Matrigel contains the relevant properties for elucidating novel phenomenon in tissue-like environments. Specifically, the gelled matrix has a high concentration of proteins, a broad range of pore sizes, and a variety of surface charges. Matrigel has been shown to have a hydraulic conductivity similar (within 21\%) to that of porcine glomular basement membrane [23]. Previous research has used Matrigel to demonstrate the significance of surface charge in mediating particle motion through biopolymers [17]. Quantitative magnetophoresis studies have been performed in Matrigel [9], and the diffusive motion of gold [24] and polymeric [17] particles has been studied in this material. Owing to its wide availability, simple preparation, and similarity to basement membranes in vivo, we chose Matrigel as a reasonable in vitro approximation of the extracellular matrix environment.

\section{Experimental Procedure}

\section{A. Nickel Nanorods}

Nickel nanorods were grown via electrodeposition into the pores of an anodized aluminum oxide membrane with $18 \mathrm{~nm}$ diameter pores (AAO, Synkera Technologies, Inc.). These membranes were first sealed on one side by thermal evaporation of a silver working electrode. After sealing one side, electroplating of nickel was performed into the pores of the template. Following nanowire synthesis, the silver working electrode was etched in dilute nitric acid, and the AAO template was etched in $0.5 \mathrm{M}$ sodium hydroxide. Detailed synthesis procedures are well documented in the literature [25]-[28]. In order to minimize adhesion between ECM proteins and nanorods, nickel nanorod surfaces were functionalized with 1 $\mathrm{kDa}$ methoxy-PEG-silane [29]. This surface functionalization effectively minimized zeta potential from an average $-46 \mathrm{mV}$ to an average $-3 \mathrm{mV}$ [21]. Previous research has shown that such narrow diameter nanorods comprise single domain particles, and will exhibit high remanence and preferential magnetization along the long axis of the nanorod [30]-[33]. 


\section{B. Magnetophoresis Experiments}

Magnetophoresis of $18 \mathrm{~nm}$ diameter nickel nanorods was assessed by mixing rods into Matrigel. Matrigel was stored at $-20^{\circ} \mathrm{C}$ prior to the experiment. The matrix was then thawed at $4{ }^{\circ} \mathrm{C}$ for sample preparation. All pipette tips and glass slides were stored at $4{ }^{\circ} \mathrm{C}$ to prevent rapid gelation during sample preparation. The nanorods were dispersed in phosphate buffered saline (PBS), chilled to $4^{\circ} \mathrm{C}$, and added to undiluted Matrigel at $1 \%$ vol./vol. A cover slip was sealed atop the sample chamber to minimize evaporation.

The composite sample (99\% Matrigel, 1\% PBS with nanorods) was gelled in an incubator for one hour $\left(37^{\circ} \mathrm{C}, 95 \%\right.$ humidity, and $\left.5 \% \mathrm{CO}_{2}\right)$, then placed on a microscope and imaged in a known magnetic field and field gradient (Fig. 2 inset). Experiments were performed at $25^{\circ} \mathrm{C}$.

The magnetic field was supplied by a calibrated, stationary, cylindrical, $\mathrm{NdFeB}$ permanent magnet.

Magnetic field strength and the equation describing field as function of distance from the magnet are shown in Fig. 2. We collected magnetophoresis data at a rate of 1 frame per second using a Pulnix PTM-6710CL camera and custom image acquisition software.

Imaging was performed in transmitted light mode using a 100x dry microscope objective. Continuous imaging was performed for tens of minutes. Videos were analyzed using Spot Tracker (freely available at cismm.org [34]). As magnetophoresis experiments were performed over the course of tens of minutes, we observed nanorod transport over distances of tens of micrometers.

\section{Results}

\section{A. Magnetophoresis and Applied Translational Force}

Unlike nanorods with larger diameters, $18 \mathrm{~nm}$ diameter nanorods experience significant acceleration and deceleration during transport. This is a consequence of their small size, the relatively small applied magnetic force, and transient steric hindrance. The force applied to a nanorod is calculated based on the analytical expression for the magnetic field as a function of distance from the magnet face and the nanorod's volume (Fig. 2) [11]. Using a $\mathrm{NdFeB}$ permanent magnet we pulled nickel nanorods with an average force of $99 \pm 27 \mathrm{fN}$ (mean \pm standard deviation). Nanorods move with an average velocity of $4.3 \pm 2.9 \mu \mathrm{m} / \mathrm{min}$. (mean standard deviation) [21].

During constant gradient magnetophoresis in Newtonian environments, the drag force $F_{\text {drag }}$ is equivalent to the magnetic force $F_{\text {magnetic }}$. In dense biopolymer environments such as the ECM, the fibrous proteins forming the matrix offer significant steric forces $F_{\text {steric }}$ which oppose the magnetic force. Electron microscopy of the matrix and particle tracking of individual rod transport suggest that $F_{\text {steric }}$ is largest at matrix protein clusters. Indeed, we observe strong steric hindrance for several minutes at a time, and calculate that these nanorods are strongly hindered approximately $94 \pm 3 \%$ of the experiment time. We define 
strong steric hindrance as a forward velocity less than $25 \%$ of the average velocity, or a forward velocity below approximately $1 \mu \mathrm{m} / \mathrm{min}$.

Single particle tracking reveals that, when averaged over time, small diameter nanorods experience near complete steric hindrance at the supplied $F_{\text {magnetic }}$. As a result, the vast majority of long-range transport is achieved via infrequent "jump" events during which the nanorod translates (comparatively) very quickly through the matrix. During large jumps nanorod velocities reached $18 \mu \mathrm{m} / \mathrm{min}$. These velocities correspond to local apparent viscosities of approximately $100 \mathrm{mPa}$.s (DI water is approximately $1 \mathrm{mPa} . \mathrm{s}$ at room temperature).

These rapid jumps indicate that other forces, such as lateral forces, torques, or forces away from the intended direction may enable significant increases in small nanorod transport through tortuous biopolymer environments by decreasing the fraction of time spent sterically confined in the matrix.

\section{B. Interactions between Nanorods}

Typical experiments were performed with low densities of nanorods (approximately $1 \times 10^{3}$ rods per $1 \mu$ sample material). Thus, nanorod-nanorod interactions were rarely observed. However, observations of these interactions proved qualitatively similar to the expected behavior in Newtonian environments: nanorods approached one another, magnetically agglomerated, then continued moving in unison. Due to the small dimension of the nanorods we were unable to resolve the exact rod-rod configuration of these pairs after agglomeration. Fig. 4 shows one such event occurring. During this event it is clear that nanorod-nanorod intra-pair magnetophoresis is moderated by matrix density: the event is a result of one nanorod making significant lateral deviations so as to eventually agglomerate with a nearby nanorod, which has been sterically hindered for several minutes.

\section{Conclusion}

In the context of magnetic drug targeting, high velocity, directed transport of nanoparticles through the tumor ECM is generally desired, as this diminishes the required time for moving drugs through the tumor volume. Thus, minimizing the amount of time nanoparticles spend sterically hindered in the matrix maximizes the potential for magnetic drug targeting. Our previous study revealed that larger diameter nanorods $(55 \mathrm{~nm}$ and $200 \mathrm{~nm}$ ) experienced constant steric hindrance and, as a result, moved at significantly lower velocities despite experiencing drastically larger forces [21]. Here we present details of the magnetophoretic motion of $18 \mathrm{~nm}$ diameter nanorods, including a quantification of the amount of time spent effectively translating versus sterically hindered, as well as the observation of intra-pair magnetophoresis in a viscoelastic medium.

\section{Acknowledgments}

The authors gratefully acknowledge the help of Wallace Ambrose of the UNC Chapel Hill Analytical and Nanofabrication Laboratory for help in preparing the Matrigel samples for SEM imaging. LOM acknowledges the Ross and Charlotte Johnson Family Dissertation Fellowship, graciously provided through the UNC Graduate School, for partially funding this research. This research was also supported by NIH \#P41EB002025. 


\section{References}

1. Pamme N. Magnetism and microfluidics. Lab Chip. 2006; 6:24-38. [PubMed: 16372066]

2. Dobson J. Magnetic micro- and nano-particle-based targeting for drug and gene delivery. Nanomedicine (Lond). 2006; 1(1):31-37. [PubMed: 17716207]

3. Thiesen B, Jordan A. Clinical applications of magnetic nanoparticles for hyperthermia. Int $\mathbf{J}$ Hyperthermia. Sep; 2008 24(6):467-74. [PubMed: 18608593]

4. Arruebo M, Fernández-pacheco R, Ibarra MR, Santamaría J. Magnetic nanoparticles for drug delivery. Nano Today. 2007; 2(3):22-32.

5. Lubbe AS, Alexiou C, Bergemann C. Clinical applications of magnetic drug targeting. J Surg Res. 2001; 95(2):200-206. [PubMed: 11162046]

6. Jang SH, Wientjes MG, Lu D, Au JLS. Drug delivery and transport to solid tumors. Pharm Res. Sep; 2003 20(9):1337-1350. [PubMed: 14567626]

7. Jain RK, Stylianopoulos T. Delivering nanomedicine to solid tumors. Nat Rev Clin Onc. Nov; 2010 7(11):653-664.

8. Overby DR, Alenghat FJ, Montoya-Zavala M, Bei H, Oh P, Karavitis J, Ingber DE. Magnetic Cellular Switches. IEEE Trans Magn. Jan; 2004 40(4):2958-2960. [PubMed: 23097592]

9. Kuhn SJ, Hallahan DE, Giorgio TD. Characterization of superparamagnetic nanoparticle interactions with extracellular matrix in an in vitro system. Ann Biomed Eng. Jan; 2006 34(1):5158. [PubMed: 16477503]

10. Kuhn SJ, Finch SK, Hallahan DE, Giorgio TD. Proteolytic surface functionalization enhances in vitro magnetic nanoparticle mobility through extracellular matrix. Nano Lett. 2006; 6(2):306-312. [PubMed: 16464055]

11. Cribb JA, Meehan TD, Shah SM, Skinner K, Superfine R. Cylinders vs.Spheres : Biofluid Shear Thinning in Driven Nanoparticle Transport. Ann Biomed Eng. 2010; 38(11):3311-3322. [PubMed: 20571853]

12. MacDonald C, Friedman G, Alamia J, Barbee K, Polyak B. Time-varied magnetic field enhances transport of magnetic nanoparticles in viscous gel. Nanomedicine (Lond). 2010; 5(1):65-76. [PubMed: 20025465]

13. Fan DL, Zhu FQ, Cammarata RC, Chien CL. High-Speed Rotation of Nanowires," Controllable. Phys Rev Lett. 2005; 247208

14. Petit T, Zhang L, Peyer KE, Kratochvil BE, Nelson BJ. Selective trapping and manipulation of microscale objects using mobile microvortices. Nano Lett. Jan; 2012 12(1):156-60. [PubMed: 22111870]

15. Kim K, Xu X, Guo J, Fan DL. Ultrahigh-speed rotating nanoelectromechanical system devices assembled from nanoscale building blocks. Nat Commun. 2014; 5:3632. [PubMed: 24709694]

16. Schuerle S, Erni S, Flink M, Kratochvil BE, Nelson BJ. Three-Dimensional Magnetic Manipulation of Micro- and Nanostructures for Applications in Life Sciences. IEEE Trans Magn. Jan; 2013 49(1):321-330.

17. Lieleg $\mathrm{O}$, Baumgärtel RM, Bausch $A R$. Selective filtering of particles by the extracellular matrix: an electrostatic bandpass. Biophys J. Sep; 2009 97(6):1569-1577. [PubMed: 19751661]

18. Lieleg O, Vladescu I, Ribbeck K. Characterization of particle translocation through mucin hydrogels. Biophys J. May; 2010 98(9):1782-1789. [PubMed: 20441741]

19. Lieleg O, Ribbeck K. Biological hydrogels as selective diffusion barriers. Trends Cell Biol. Sep; 2011 21(9):543-551. [PubMed: 21727007]

20. Kirch J, Schneider A, Abou B, Hopf A, Schaefer UF, Schneider M, Schall C, Wagner C, Lehr CM. Optical tweezers reveal relationship between microstructure and nanoparticle penetration of pulmonary mucus. Proc Natl Acad Sci U S A. Nov; 2012 109(45):18355-60. [PubMed: 23091027]

21. Mair LO, Superfine R. Single particle tracking reveals biphasic transport during nanorod magnetophoresis through extracellular matrix. Soft Matter. Apr.2014 10:4118-4125. [PubMed: 24744160] 
22. Yurchenco PD, Ruben GC. Type IV Collagen Lateral Associations in the EHS Tumor Matrix Comparison with Amniotic and In Vitro Networks. Am J Pathol. 1988; 132(2):278-291. [PubMed: 3400773]

23. Katz MA, Barrette T, Krasovich M. Hydraulic conductivity of basement membrane with computed values for fiber radius and void volume. Am J Physiol Hear Circ Physiol. 1998; 274(4):H1327H1334.

24. Kim B, Han G, Toley BJ, Kim CK, Rotello VM, Forbes NS. Tuning payload delivery in tumour cylindroids using gold nanoparticles. Nat Nanotechnol. 2010; 5(6):465-472. [PubMed: 20383126]

25. Penner RM, Martin CR. Preparation and electrochemical characterization of ultramicroelectrode ensembles. Anal Chem. Nov; 1987 59(21):2625-2630.

26. Martin CR. Nanomaterials: A Membrane-Based Approach Synthetic Approach. Science (80- ). 1994; 266(5193):1961-1966.

27. Fert A, Piraux L. Magnetic nanowires. J Magn Magn Mater. Oct; 1999 200(1-3):338-358.

28. Sharma A, Zhu Y, Thor S, Zhou F, Stadler B, Hubel A, Fabrication AN. Magnetic Barcode Nanowires for Osteosarcoma Cell Control, Detection and Separation. IEEE Trans Magn. 2013; 49(1):453-456.

29. Zhang Y, Kohler N, Zhang M. Surface modification of superparamagnetic magnetite nanoparticles and their intracellular uptake. Biomaterials. 2002; 23:1553-1561. [PubMed: 11922461]

30. Nielsch K, Wehrspohn RB, Barthel J, Krischner J, Gösele U, Fischer SF, Kronmüller H. Hexagonally ordered $100 \mathrm{~nm}$ period nickel nanowire arrays. Appl Phys Lett. 2001; 79(9):1360.

31. Ross C, Hwang M, Shima M, Cheng J, Farhoud M, Savas T, Smith H, Schwarzacher W, Ross F, Redjdal M, Humphrey F. Micromagnetic behavior of electrodeposited cylinder arrays. Phys Rev B. Mar.2002 65(14):144417.

32. Zeng H, Skomski R, Menon L, Liu Y, Bandyopadhyay S, Sellmyer D. Structure and magnetic properties of ferromagnetic nanowires in self-assembled arrays. Phys Rev B. Mar.2002 65(13): 134426.

33. Günther A, Monz S, Tschöpe A, Birringer R, Michels A. Angular dependence of coercivity and remanence of Ni nanowire arrays and its relevance to magnetoviscosity. J Magn Magn Mater. Apr; 2008 320(7):1340-1344.

34. Taylor, RM. Video Spot Tracker software, Version 6.04. 2009. online at http://cismm.cs.unc.edu/ downloads/?dl_cat=3 


\section{Highlights}

- We study the magnetophoretic transport of $18 \mathrm{~nm}$ diameter nickel nanorods through a protein-rich matrix.

- We note that nanorods move with low velocity (less than 1 micrometer per minute) for approximately $95 \%$ of the total experiment time.

- We plot nanorod velocity over the course of hundreds of seconds.

- Finally, we observe intra-particle magnetophoresis events in this viscoelastic environment. 

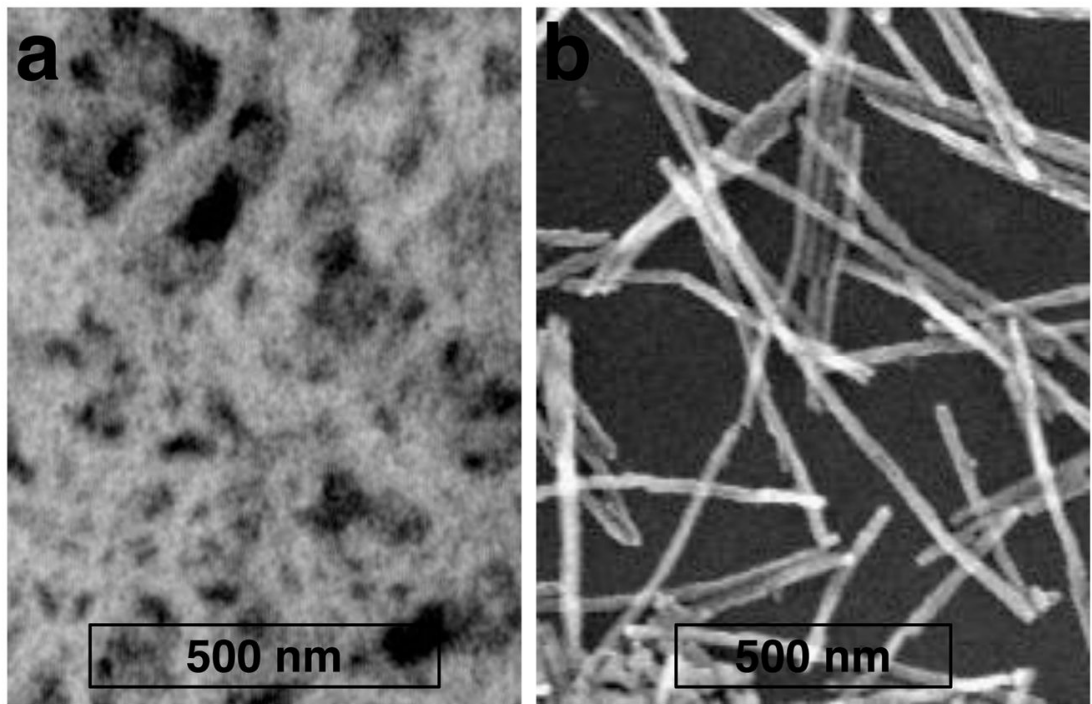

Fig. 1.

Scanning electron microscope images of (a) Matrigel and (b) electrodeposited $18 \mathrm{~nm}$ diameter nickel nanorods. 


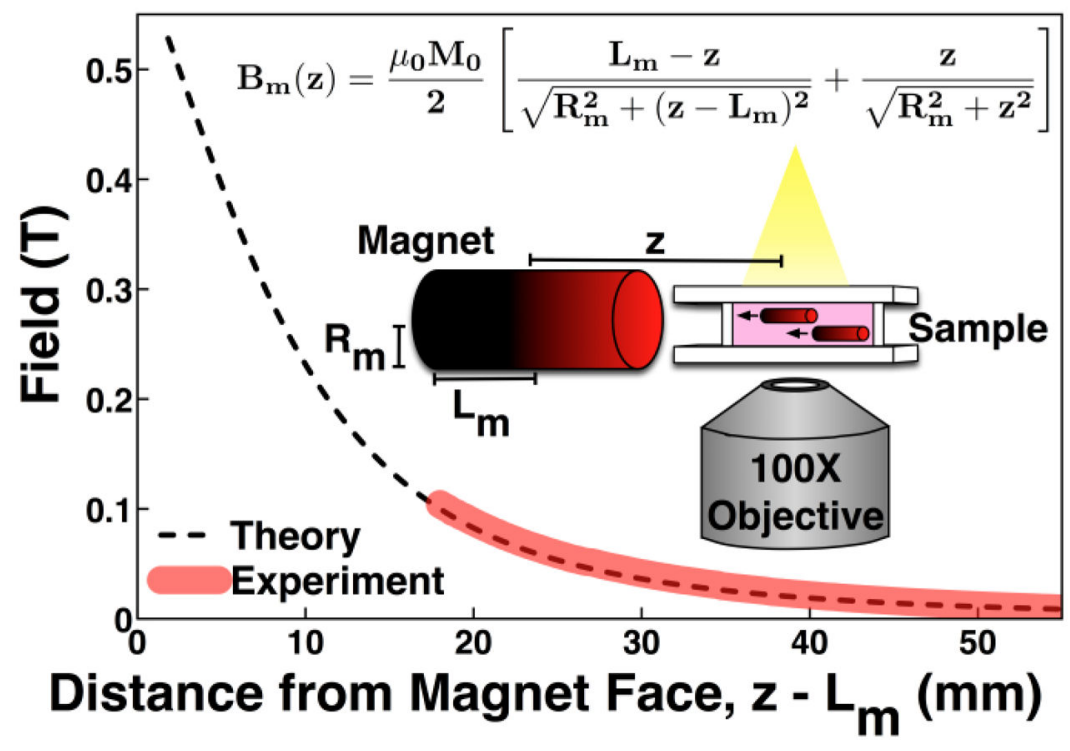

Fig. 2.

Measured magnetic field $(\mathrm{T})$ as a function of distance $(\mathrm{mm})$ from the face of the cylindrical permanent magnet is shown (solid red line). Field measurements are in $0.25 \mathrm{~mm}$ increments. The theoretical equation for the magnetic field as a function of distance from the face of a cylindrical magnet is shown (top) and plotted (dashed black line). The experimental setup consisting of the pulling magnet, illumination, nanorod sample, and microscope objective is depicted (not to scale). The parameters $\mathrm{R}_{\mathrm{m}}$ (radius of the magnet), $\mathrm{L}_{\mathrm{m}}$ (one half the length of the magnet), and $\mathrm{z}$ (distance between nanorod and magnet center) are shown. $\mathrm{B}_{\mathrm{m}}(\mathrm{z})$ is the magnetic field, $\mu_{0}$ is the permeability of free space, and $M_{0}$ is the magnetic saturation of the magnet. 


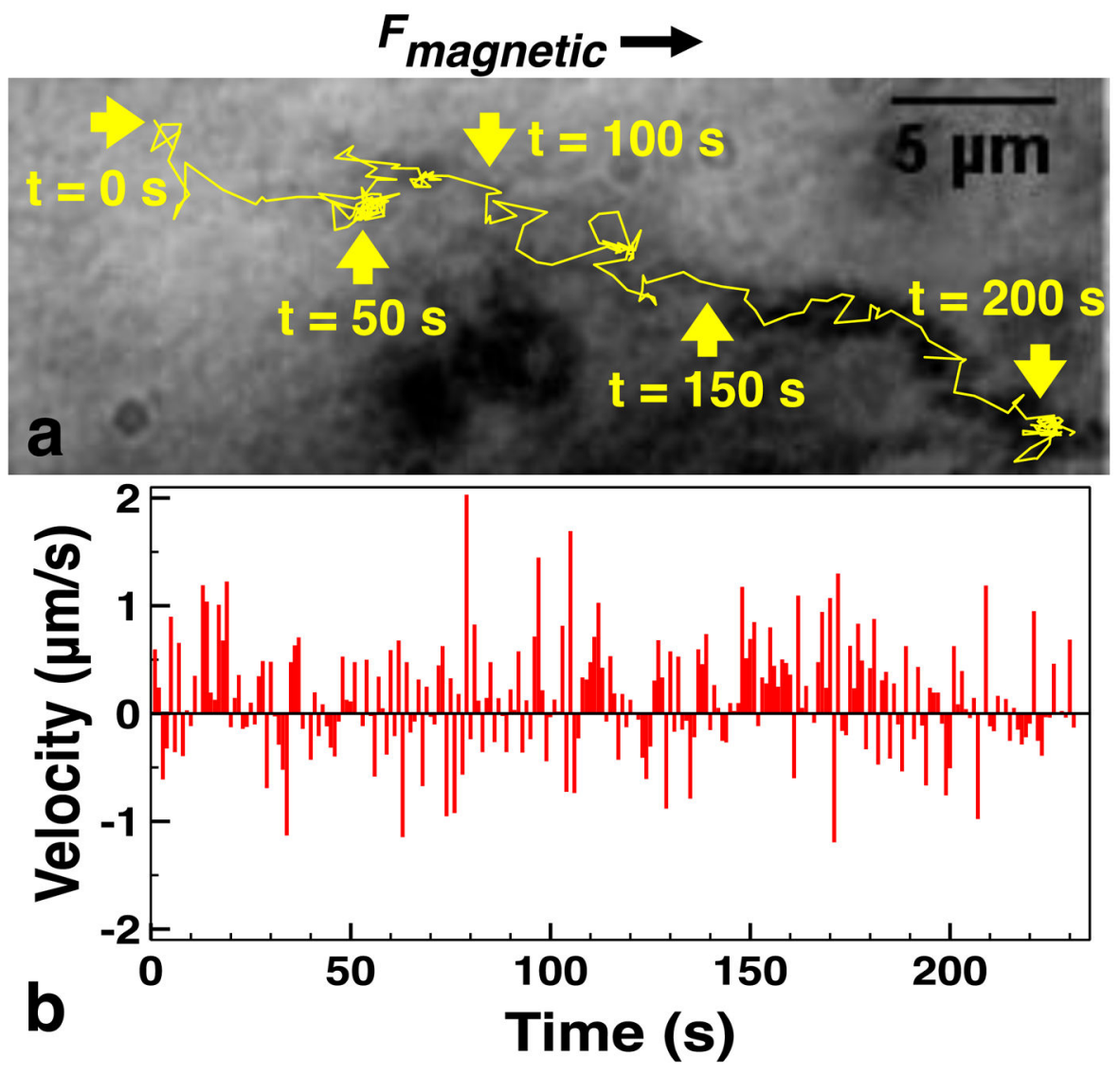

Fig. 3.

(a) Traces of nanorod motion (yellow line) overlaid on a minimum intensity projection of nanorod transport through Matrigel. Arrows indicate time (seconds). Dark regions in the center of the image (below the nanorod path trace) are due to Matrigel inhomogeneity. As (a) is a minimum intensity projection of 232 images, density variations in the Matrigel appear exaggerated. (b) Nanorod translational velocity in the direction of $F_{\text {magnetic }}$. The applied gradient pulls the nanorod from left to right. 

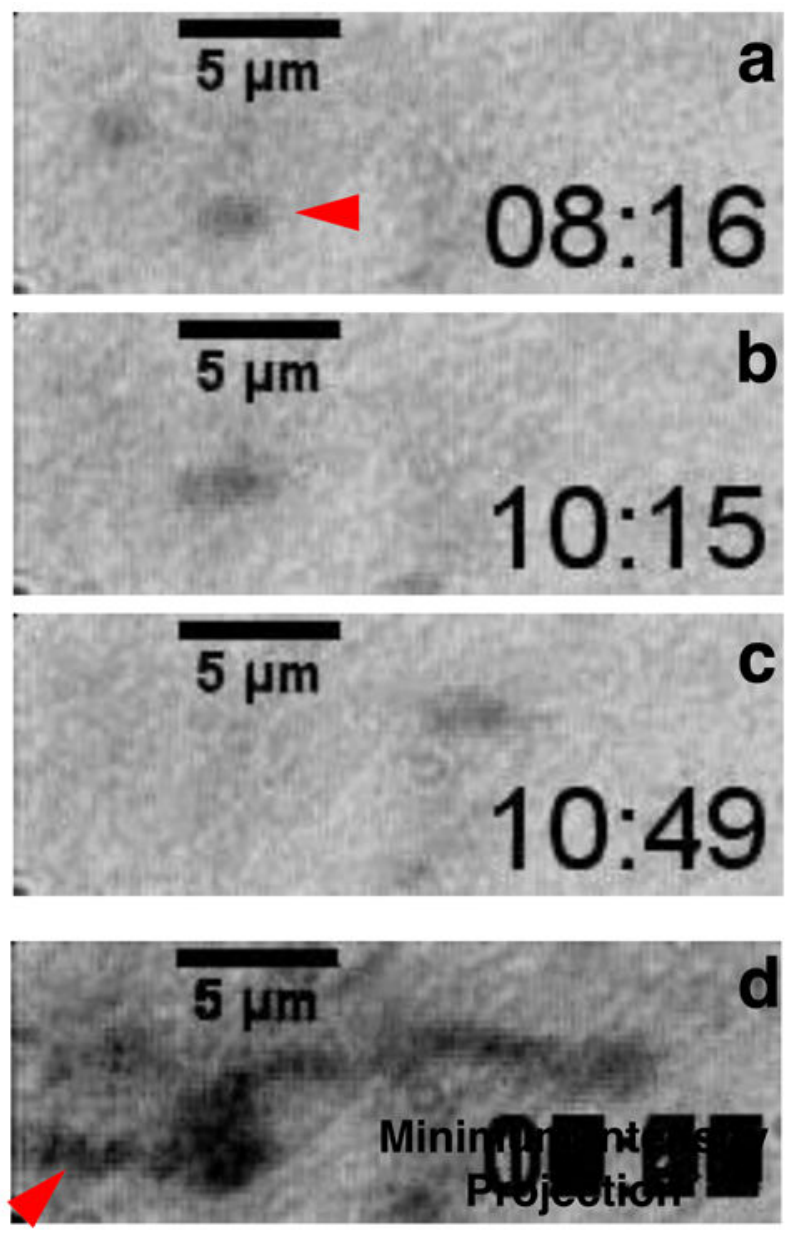

Fig. 4.

Nanorod-nanorod intra-pair magnetophoresis (a), resulting agglomeration (b), and subsequent motion (c). The magnetic force moves particles from left to right. Agglomerated nanorods also demonstrated acceleration and deceleration, indicating extreme steric hindrance was a transient property for these agglomerations. (a) Two separate nanorods, indicated by triangles. (b) Agglomeration of nanorods due to intra-pair magnetophoretic forces. (c) Continued magnetophoretic motion. (d) A minimum intensity projection of all movie frames demonstrates the paths taken by the two nanorods prior to agglomeration (triangles), as well as their unified course after agglomeration. Time stamp is in minutes:seconds. 